\title{
Classification of Simple $W^{\prime}$ Models
}

\section{Tomohiro Abe*}

Institute for Advanced Research, Nagoya University, Furo-cho Chikusa-ku, Nagoya, Aichi, 464-8602 Japan

Kobayashi-Maskawa Institute for the Origin of Particles and the Universe, Nagoya University,

Furo-cho Chikusa-ku, Nagoya, Aichi, 464-8602 Japan

E-mail: abetomo@kmi.nagoya-u.ac.jp

\section{Ryo Nagai}

Department of Physics, Nagoya University, Furo-cho Chikusa-ku, Nagoya, Aichi, 464-8602

Japan

E-mail: nagai@th.phys.nagoya-u.ac.jp

\begin{abstract}
We investigate decay modes of spin-1 heavy vector bosons $\left(V^{\prime}\right)$ from the viewpoint of perturbative unitarity in a model independent manner. Perturbative unitarity requires some relations among couplings, which are called unitarity sum rules. We derive the relation between $V^{\prime}$ couplings to the SM fermions $(f)$ and $V^{\prime}$ couplings to the SM gauge bosons $(V)$. Using the coupling relations, we calculate partial decay widths for $V^{\prime}$ decays into $V V$ and $f f$. We show that $\operatorname{Br}\left(W^{\prime} \rightarrow W Z\right)<0.02$ in the system that contains $V^{\prime}$ and CP-even scalars as well as the SM particles. This result is independent of the number of the CP-even scalars. We also discuss what kind of interactions can make $\operatorname{Br}\left(V^{\prime} \rightarrow V V\right)$ larger than $\operatorname{Br}\left(V^{\prime} \rightarrow f f\right)$. Our result is a useful guideline to construct models that predict $\operatorname{Br}\left(W^{\prime} \rightarrow W Z\right)>0.02$.
\end{abstract}

The 3rd International Symposium on "Quest for the Origin of Particles and the Universe" 5-7 January 2017

Nagoya University, Japan

${ }^{*}$ Speaker. 


\section{Introduction}

Spin-1 heavy vector particles $\left(V^{\prime}\right)$ are popular particles predicted in physics beyond the standard model (BSM). An efficient way to study $V^{\prime}$ phenomenology at the LHC is to use effective Lagrangians. For example, the effective Lagrangian given in Ref. [1,2] provides a simple framework for $V^{\prime}$ phenomenology at the LHC, and the framework is used in the analysis of ATLAS and CMS $[3,4,5,6,7,8]$. On the other hand, the effective Lagrangian violates perturbative unitarity at higher energy scale in general. If the unitarity violation scale is as low as $\mathrm{TeV}$ scale, we have to take into account a lot of higher dimentional operators. The higher dimensional operators accompany unknown coefficients. It makes difficult to give a definite prediction due to a number of unknown coefficients.

We can avoid the perturbative unitarity violation if the Lagrangian is renormalizable. The effective Lagrangian given in Ref. [1, 2] includes a renormalizable model called HVT model A in special regions of parameter space. This model predicts that $V^{\prime}$ mainly decay into two SM fermions $(f)$ and the decay mode into two SM gauge bosons $(V)$ are much smaller, $\operatorname{Br}\left(V^{\prime} \rightarrow V V\right) \ll$ $\operatorname{Br}\left(V^{\prime} \rightarrow f f\right)$. However, we do not know the main decay mode of $V^{\prime}$ in advance of the discovery. $V^{\prime}$ could be discovered in $V V$ decay mode in future. It is thus important to prepare for the discovery in any decay modes, and thus we should prepare other Lagrangians that predict $\operatorname{Br}\left(V^{\prime} \rightarrow V V\right) \geq$ $\operatorname{Br}\left(V^{\prime} \rightarrow f f\right)$ without violating perturbative unitarity.

We investigate decay modes of spin-1 heavy vector bosons from the viewpoint of perturbative unitarity in a model independent manner. Our purpose is to figure out the conditions that $\operatorname{Br}\left(V^{\prime} \rightarrow\right.$ $V V) \geq \operatorname{Br}\left(V^{\prime} \rightarrow f f\right)$ without relying on specific models. To this purpose, we need to know the $V^{\prime}$ couplings to the gauge bosons and to the fermions. We can find coupling relations by imposing perturbative unitarity on scattering amplitudes that contain both the gauge bosons and the fermions. The relations are called unitarity sum rules. Using the unitarity sum rules, we can obtain the coupling relations to calculate $\operatorname{Br}\left(V^{\prime} \rightarrow V V\right)$ and $\operatorname{Br}\left(V^{\prime} \rightarrow f f\right)$. The unitarity sum rules depend on the matter contents of the system, and thus we can understand what kind of matter contents and interactions can make $\operatorname{Br}\left(V^{\prime} \rightarrow V V\right)$ larger than $\operatorname{Br}\left(V^{\prime} \rightarrow f f\right)$ from the unitarity sum rules.

\section{Unitarity sum rules for $f_{1} \bar{f}_{2} \rightarrow V_{3}^{-} V_{4}^{+}$}

We calculate the scattering amplitude of $f_{1} \bar{f}_{2} \rightarrow V_{3}^{-} V_{4}^{+}$denoted by $i \mathscr{M}_{j k}$, where $j$ and $k$ are the twice of the helicity of $f_{1}$ and $\bar{f}_{2}$, respectively. We include all the SM fermions, the SM gauge bosons $\left(W^{ \pm}, Z, \gamma\right)$, new heavy vector bosons $\left(W^{\prime \pm}, Z^{\prime}\right)$, CP-even scalars ( $h$ 's), and CP-odd scalars $\left(\Delta^{0}\right.$ 's) in our analysis. We assume CP conservation in the scalar sector. We also assume the minimal flavour violation (MFV) [9] for simplicity. Under these assumptions, we calculate the amplitude of $f_{1} \bar{f}_{2} \rightarrow V_{3}^{-} V_{4}^{+}$in the high energy limit,

$$
\begin{aligned}
& \mathscr{M}_{\mp \pm}=\frac{s}{2 m_{V_{3}} m_{V_{4}}} \mathscr{A}_{\mp \pm} \sin \theta+\mathscr{O}\left(s^{0}\right), \\
& \mathscr{M}_{ \pm \pm}=\frac{\sqrt{s}}{2 m_{V_{3}} m_{V_{4}}}\left(\mathscr{C}_{ \pm \pm}^{(0)}+\mathscr{C}_{ \pm \pm}^{(1)} \cos \theta+\mathscr{O}\left(s^{0}\right)\right),
\end{aligned}
$$

and we obtain unitarity sum rules by imposing $\mathscr{A}_{-+}=\mathscr{A}_{+-}=\mathscr{C}_{++}^{(0)}=\mathscr{C}_{--}^{(0)}=0$. This condition is automatically satisfied in renormalizable models. Effective $V^{\prime}$ models with sufficiently high cut-off 
scale also satisfy this condition in good approximation. If $\mathscr{A}_{-+}=\mathscr{A}_{+-}=0$, then $\mathscr{C}_{++}^{(1)}$ and $\mathscr{C}_{--}^{(1)}$ are automatically equal to zero. The sum rules from $\mathscr{C}_{++}^{(0)}$ and $\mathscr{C}_{--}^{(0)}$ contain both the CP-even and CP-odd couplings. We can separate these couplings by taking linear combinations, $\mathscr{C}_{++}^{(0)} \pm \mathscr{C}_{--}^{(0)}$. In the following sections, we apply the sum rules to two simple setups and discuss the relation between $\Gamma\left(W^{\prime} \rightarrow W Z\right)$ and $\Gamma\left(W^{\prime} \rightarrow f f\right)$.

\section{3. $\mathbf{S M}+V^{\prime}+\mathbf{C P}$-even scalars}

In this section, we apply the unitarity sum rules to the following simple setup. We consider $\mathrm{SU}(2)_{0} \times \mathrm{SU}(2)_{1} \times \mathrm{U}(1)_{2}$ electroweak gauge symmetry. Left-handed fermions are $\mathrm{SU}(2)_{1}$ doublets. Right-handed fermions are singlet under both $\mathrm{SU}(2)_{0}$ and $\mathrm{SU}(2)_{1}$. Both the left- and right-handed fermions have appropriate $\mathrm{U}(1)_{2}$ charge. This charge assignment implies that the charged gauge bosons do not couple to the right-handed currents. All scalars are CP-even. We do not include $\mathrm{CP}$-odd scalars in the setup here. We do not specify the number of the CP-even scalars. This setup contains HVT model A [1,2]. Under this setup, we obtain the $u \bar{u} \rightarrow V_{3}^{-} V_{4}^{+}$unitarity sum rules as follows.

$$
\begin{aligned}
\frac{1}{2} g_{V_{3}} g_{V_{4}} & =\sum_{V=\gamma, Z, Z^{\prime}} g_{V_{3}^{+} V_{4}^{-} V} g_{\bar{u} u V}^{L}, \\
0 & =\sum_{V=\gamma, Z, Z^{\prime}} g_{V_{3}^{+} V_{4}^{-} V} g_{\bar{u} u V}^{R}, \\
g_{V_{3}} g_{V_{4}} & =2 \sum_{h} g_{V_{3} V_{4} h} \frac{g_{\bar{u} u h}}{m_{u}}, \\
0 & =\sum_{V=Z, Z^{\prime}} \frac{m_{V_{3}}^{2}-m_{V_{4}}^{2}}{m_{V}^{2}} g_{V_{3}^{+} V_{4}^{-} V}\left(g_{\bar{u} u V}^{L}-g_{\bar{u} u V}^{R}\right) .
\end{aligned}
$$

Combining these unitarity sum rules, we can erase $g_{W W^{\prime} Z^{\prime}}$ and obtain

$$
g_{W W^{\prime} Z}=-\frac{m_{Z}^{2}}{m_{Z^{\prime}}^{2}} \frac{g_{W} g_{W^{\prime}}}{2\left(g_{\bar{u} u Z}^{L}-g_{\bar{u} u Z}^{R}\right)} \frac{1}{1-\frac{m_{Z}^{2}}{m_{Z^{\prime}}^{2}}} .
$$

In general, $g_{W}, g_{\bar{u} u Z}^{L}$, and $g_{\bar{u} u Z}^{R}$ are different from the SM prediction but should become the same as in the SM in the decoupling limit $\left(m_{W^{\prime}, Z^{\prime}} \rightarrow \infty\right)$. Thus the relation among $g_{W}, g_{\bar{u} u Z}^{L}, g_{\bar{u} u Z}^{R}, m_{W}$, and $m_{Z}$ are the same as in the SM at the leading order in the large $m_{W^{\prime}, Z^{\prime}}$ limit. In a similar manner, we obtain the $\ell \bar{\ell} \rightarrow W^{-} W^{\prime+}$ perturbative unitarity sum rules. We find

$$
g_{W W^{\prime} Z} \simeq-\frac{m_{W^{\prime}} m_{Z}}{m_{Z^{\prime}}^{2}} g_{W^{\prime}} \simeq-\frac{m_{W} m_{Z}}{m_{Z^{\prime}}^{2}} g_{W^{\prime}}^{\ell}
$$

We can see that $g_{W^{\prime}}^{\ell} \simeq g_{W^{\prime}}$, and thus the relation given in Eq. (3.6) is flavor independent. From these coupling relations, we find the following ratios of partial widths for the $W^{\prime}$ decays into $W Z$ and $f f$,

$$
\frac{\Gamma\left(W^{\prime} \rightarrow W Z\right)}{\Gamma\left(W^{\prime} \rightarrow f_{i} f_{j}\right)} \simeq \frac{1}{4 c_{i j}}
$$


where

$$
c_{i j}=\left\{\begin{array}{ll}
N_{c}\left|V_{C K M}^{i j}\right|^{2} & \text { (for quarks) } \\
\delta^{i j} & \text { (for leptons) }
\end{array},\right.
$$

where $N_{c}=3$, and the terms of order $m_{W, Z, f}^{2} / m_{W^{\prime}}^{2}$ have been neglected. Here we assumed $m_{W^{\prime}}^{2} \simeq m_{Z^{\prime}}^{2}$ to avoid the large custodial SU(2) symmetry breaking. The important consequence of Eq. (3.7) is the suppression of $\operatorname{Br}\left(W^{\prime} \rightarrow W Z\right)$. Since there are three generations in both lepton and quark sectors,

$$
\frac{\Gamma\left(W^{\prime} \rightarrow W Z\right)}{\sum_{f} \Gamma\left(W^{\prime} \rightarrow f_{i} f_{j}\right)} \simeq \frac{1}{4 \times\left(\left(N_{c}+1\right) \times 3\right)}=\frac{1}{48},
$$

where we used $\sum_{i, j}\left|V_{C K M}^{i j}\right|^{2}=3$. This equation implies that $\operatorname{Br}\left(W^{\prime} \rightarrow W Z\right) \lesssim 2 \%$. If we assume the equivalent theorem relation $\Gamma\left(W^{\prime} \rightarrow W Z\right) \simeq \Gamma\left(W^{\prime} \rightarrow W h\right)$ and the $W^{\prime}$ decay to $W$ and heavy neutral scalar is highly suppressed, we find $\operatorname{Br}\left(W^{\prime} \rightarrow W Z\right) \simeq 2 \%$ and $\operatorname{Br}\left(W^{\prime} \rightarrow e v\right) \simeq 8 \%\left(\sum_{f} \operatorname{Br}\left(W^{\prime} \rightarrow f f\right)\right.$ $\simeq 96 \%$ ). The assumption is justified in the case where $g_{W W h} \simeq g_{W W h}^{\mathrm{SM}}[10]$. Therefore, the branching ratio of $W^{\prime}$ to the gauge bosons is much smaller than to the fermions. This result has been derived from the $f \bar{f} \rightarrow W W^{\prime}$ unitarity sum rules and does not need perturbative unitarity of other processes such as $W W \rightarrow W W$. In addition, the result does not depend on the number of the CP-even scalars. Therefore our result in this section can be applied to various models.

If $\operatorname{Br}\left(W^{\prime} \rightarrow W Z\right)$ is measured in future and if it is larger than $2 \%$, it is implied that perturbative unitarity of $f \bar{f} \rightarrow W W^{\prime}$ is violated or the other new particles in addition to $W^{\prime}, Z^{\prime}$ and CP-even scalar bosons exist.

In this section we have assumed $g_{W^{\prime}} \neq 0$. If $g_{W^{\prime}}=0$, then $g_{W W^{\prime} Z}=0$ as we can see from Eq. (3.5), and $W^{\prime}$ decouples from the SM sector if $g_{W^{\prime}}=0$.

\section{4. $\mathbf{S M}+V^{\prime}+\mathbf{C P}$-even scalars + CP-odd scalars}

We extend the analysis in the previous section by introducing CP-odd scalars, and show that $\operatorname{Br}\left(W^{\prime} \rightarrow W Z\right)$ can become larger than $\operatorname{Br}\left(W^{\prime} \rightarrow f f\right)$. The unitarity sum rule in Eq. (3.4) is modified by the contributions of the CP-odd scalars. Instead of Eq. (3.4), we obtain the following unitarity sum rule.

$$
\sum_{\Delta^{0}} \frac{g_{\bar{u} u \Delta^{0}}}{m_{u}} g_{V_{3} V_{4} \Delta^{0}}=\sum_{V=Z, Z^{\prime}} 2 g_{V_{3} V_{4} V} \frac{m_{V_{3}}^{2}-m_{V_{4}}^{2}}{m_{V}^{2}}\left(g_{\bar{u} u Z}^{L}-g_{\bar{u} u Z}^{R}\right) .
$$

The difference of this equation from Eq. (3.4) is that the left-hand side can be nonzero because of the contributions of the CP-odd scalars. This is the only difference of the sum rules in this section from the previous section. This difference can make $\Gamma\left(W^{\prime} \rightarrow W Z\right)$ change drastically as we will see in the following. Using Eqs. (3.1), (3.2), (4.1), and similar unitarity sum rules from the amplitude for $\ell \bar{\ell} \rightarrow W^{-} W^{\prime+}$, we find that

$$
\begin{aligned}
& g_{W W^{\prime} Z} \simeq-\frac{m_{W} m_{Z}}{m_{Z^{\prime}}^{2}}\left(g_{W^{\prime}}+x_{\Delta}\right), \quad \text { where } x_{\Delta}=\sum_{\Delta} \frac{g_{\bar{u} u \Delta}}{m_{u}} \frac{g_{W W^{\prime} \Delta}}{g_{W}} ., \\
& g_{W W^{\prime} Z} \simeq-\frac{m_{W^{\prime}} m_{Z}}{m_{Z^{\prime}}^{2}}\left(g_{W^{\prime}}^{\ell}+x_{\Delta}^{\ell}\right), \quad \text { where } x_{\Delta}^{\ell}=\sum_{\Delta} \frac{g_{\bar{\ell} \ell \Delta}}{m_{\ell}} \frac{g_{W W^{\prime} \Delta}}{g_{W}^{\ell}} .
\end{aligned}
$$


Here we have neglected the terms of order $m_{W, Z, f}^{2} / m_{W^{\prime}}^{2}$ and we assumed that $m_{W^{\prime}} \simeq m_{Z^{\prime}}$. Unlike the setup discussed in Sec. 3, we cannot conclude $g_{W^{\prime}}^{\ell} \simeq g_{W^{\prime}}$ in this setup. We have two comments on Eq. (4.2). First, this equation is independent of the quark flavor, although $x_{\Delta}$ looks quark flavor dependent. This is because $x_{\Delta} \simeq-g_{W^{\prime}}-g_{W W^{\prime} Z} m_{Z^{\prime}}^{2} / m_{W} m_{Z}$ and the right-hand side of this equation is independent of quark flavor. Second, Eq. (4.2) implies that $g_{W W^{\prime} Z} \neq 0$ even if $g_{W^{\prime}}=0$ as long as the CP-odd couplings exist. In the case where $g_{W^{\prime}}=0$ and $g_{W W^{\prime} Z} \neq 0$, the $W^{\prime}$ decay to $W Z$ can be the dominant decay mode. This is a big difference of the current setup from the setup discussed in Sec. 3 .

Using Eqs. (4.2) and (4.3), we find

$$
\begin{aligned}
\frac{\Gamma\left(W^{\prime} \rightarrow W Z\right)}{\Gamma\left(W^{\prime} \rightarrow u_{i} d_{j}\right)} \simeq \frac{\left(g_{W^{\prime}}+x_{\Delta}\right)^{2}}{4 N_{c}\left|V_{C K M}^{i j}\right|^{2} g_{W^{\prime}}^{2}}, \quad \frac{\Gamma\left(W^{\prime} \rightarrow W Z\right)}{\Gamma\left(W^{\prime} \rightarrow \ell v\right)} \simeq \frac{\left(g_{W^{\prime}}^{\ell}+x_{\Delta}^{\ell}\right)^{2}}{4\left(g_{W^{\prime}}^{\ell}\right)^{2}}, \\
\frac{\Gamma\left(W^{\prime} \rightarrow W Z\right)}{\sum \Gamma\left(W^{\prime} \rightarrow f_{i} f_{j}\right)} \simeq\left(4 N_{c} \frac{3}{\left(1+\frac{x_{\Delta}}{g_{W^{\prime}}}\right)^{2}}+4 \frac{3}{\left(1+\frac{x_{\Delta}^{\ell}}{g_{W^{\prime}}^{\ell}}\right)^{2}}\right)^{-1},
\end{aligned}
$$

where the terms of order $m_{W, Z, f}^{2} / m_{W^{\prime}}^{2}$ have been neglected. The factor three in Eq. (4.5) is the number of the generation. We used $\sum_{i, j}\left|V_{C K M}^{i j}\right|^{2}=3$.

We find that $g_{W W^{\prime} Z}$ and $\Gamma\left(W^{\prime} \rightarrow W Z\right)$ depend on $g_{W^{\prime}}, x_{\Delta}$, and $x_{\Delta}^{\ell}$. This dependence is a different feature from Eqs. (3.7) and (3.9). If the CP-odd scalars are absent, then the ratio of the two partial decay widths is uniquely determined as we have discussed in Sec. 3, see Eq. (3.9). On the other hand, in the system with the CP-odd scalars, the ratio of the two partial decay widths is controlled by $g_{W^{\prime}}, x_{\Delta}$, and $x_{\Delta}^{\ell}$, which are model dependent parameters controlled by the CP-odd scalar couplings. Thanks to this feature, $\Gamma\left(W^{\prime} \rightarrow W Z\right)$ can be comparable to or even larger than the other decay modes. For example, $\Gamma\left(W^{\prime} \rightarrow W Z\right) \simeq \Gamma\left(W^{\prime} \rightarrow \ell v\right)$ if $x_{\Delta}^{\ell} \simeq g_{W^{\prime}}$ or $\simeq-3 g_{W^{\prime}} . \Gamma\left(W^{\prime} \rightarrow W Z\right)$ is larger than $\Gamma\left(W^{\prime} \rightarrow f f\right)$ in large $\left|x_{\Delta} / g_{W^{\prime}}\right|$ regime. $\Gamma\left(W^{\prime} \rightarrow W Z\right)$ also can become small and even vanish for $x_{\Delta} \simeq x_{\Delta}^{\ell} \simeq-g_{W^{\prime}}$. In any case, the contributions of the CP-odd scalars significantly change the ratio of $\Gamma\left(W^{\prime} \rightarrow W Z\right)$ to $\Gamma\left(W^{\prime} \rightarrow f f\right)$ from the prediction without the CP-odd scalars. In particular, it is the important feature that $W^{\prime} \rightarrow f f$ is highly suppressed and $W^{\prime} \rightarrow W Z$ can be the dominant decay mode in this setup with large $\left|x_{\Delta} / g_{W^{\prime}}\right|$. This is the very different feature from the setup in Sec. 3.

We estimate the maximum value of $\operatorname{Br}\left(W^{\prime} \rightarrow e v\right)$ as follows.

$$
\begin{aligned}
\operatorname{Br}\left(W^{\prime} \rightarrow e v\right) & =\frac{\Gamma\left(W^{\prime} \rightarrow e v\right)}{\Gamma\left(W^{\prime} \rightarrow W Z\right)+\sum_{f} \Gamma\left(W^{\prime} \rightarrow f f\right)+\sum_{X} \Gamma\left(W^{\prime} \rightarrow X\right)} \\
& \leq \frac{\Gamma\left(W^{\prime} \rightarrow e v\right)}{\Gamma\left(W^{\prime} \rightarrow W Z\right)+\sum_{f} \Gamma\left(W^{\prime} \rightarrow f f\right)} \\
& \simeq \frac{4}{\left(1+\frac{x_{\Delta}^{\ell}}{g_{W^{\prime}}^{\ell}}\right)^{2}}\left(1+\frac{36}{\left(1+\frac{x_{\Delta}}{g_{W^{\prime}}}\right)^{2}}+\frac{12}{\left(1+\frac{x_{\Delta}^{\ell}}{g_{W^{\prime}}^{\ell}}\right)^{2}}\right)^{-1} \\
& \equiv \operatorname{Br}_{\max }\left(W^{\prime} \rightarrow e v\right),
\end{aligned}
$$

where $\Gamma\left(W^{\prime} \rightarrow X\right)$ is the sum of the other partial decay widths of $W^{\prime}$ such as $\Gamma\left(W^{\prime} \rightarrow W h\right), \Gamma\left(W^{\prime} \rightarrow\right.$ $\left.W \Delta^{0}\right) . \operatorname{Br}_{\max }\left(W^{\prime} \rightarrow e v\right)$ corresponds to the value of $\operatorname{Br}\left(W^{\prime} \rightarrow e v\right)$ assuming $\Gamma\left(W^{\prime} \rightarrow X\right)=0$. 


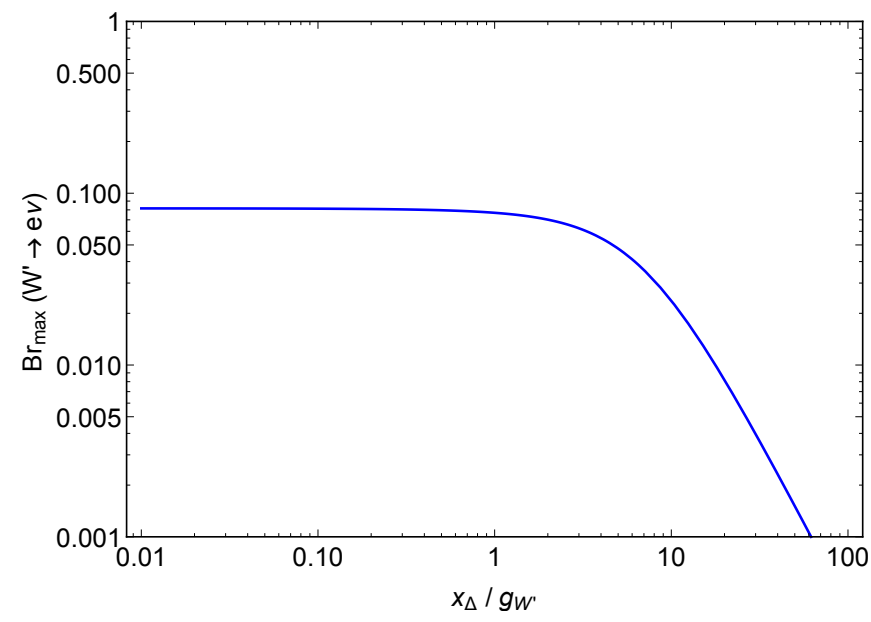

Figure 1: $\mathrm{Br}_{\max }\left(W^{\prime} \rightarrow e v\right)$ as a function of $x_{\Delta} / g_{W^{\prime}}$.

Although $\Gamma\left(W^{\prime} \rightarrow X\right)$ generally takes non-zero value in the case where $m_{W^{\prime}}>m_{X}, \operatorname{Br}\left(W^{\prime} \rightarrow e v\right)$ is always smaller than $\mathrm{Br}_{\max }\left(W^{\prime} \rightarrow e v\right)$ for any $x_{\Delta} / g_{W^{\prime}}, x_{\Delta}^{l} / g_{W^{\prime}}$, and $m_{X}$. Figure 1 shows $\mathrm{Br}_{\max }\left(W^{\prime} \rightarrow\right.$ $e v)$ as a function of $x_{\Delta} / g_{W^{\prime}}$ with assuming $x_{\Delta}^{\ell}=x_{\Delta}$ and $g_{W^{\prime}}^{\ell}=g_{W^{\prime}}$ for simplicity. We find that $\operatorname{Br}\left(W^{\prime} \rightarrow e v\right)$ is highly suppressed and $\operatorname{Br}\left(W^{\prime} \rightarrow W Z\right)$ can be large in the case with large $x_{\Delta} / g_{W^{\prime}}$. We can also see two extreme cases easily from this figure. One is $x_{\Delta} \rightarrow 0$ limit where $\operatorname{Br}_{\max }\left(W^{\prime} \rightarrow\right.$ $e v) \simeq 8 \%$. This result is consistent with the result in Sec. 3. The other case is $g_{W^{\prime}} \rightarrow 0$ limit where $\operatorname{Br}\left(W^{\prime} \rightarrow f f\right)=0$ and $W^{\prime}$ to $W Z$ can be the main decay mode. This result is again consistent with our discussion below Eq. (4.2). Negative $x_{\Delta}$ can also make $\operatorname{Br}\left(W^{\prime} \rightarrow e v\right)$ extremely small. By measuring $\operatorname{Br}\left(W^{\prime} \rightarrow e v\right)$ and using Eqs. (4.2), (4.3), and (4.6), we can estimate $x_{\Delta}$, and can obtain information of the CP-odd scalar couplings even before the discovery of $\Delta^{0}$.

The existence of the CP-odd scalar couplings is important to increase $\operatorname{Br}\left(W^{\prime} \rightarrow W Z\right)$ because $x_{\Delta}$ and $x_{\Delta}^{\ell}$ can be zero if the CP-odd scalars do not couple to the fermions or the gauge bosons as can be seen from Eqs. (4.2) and (4.3). Both $g_{\bar{f} f \Delta}$ and $g_{W W^{\prime} \Delta^{0}}$ need to be nonzero for making $\operatorname{Br}\left(W^{\prime} \rightarrow W Z\right)$ larger than $2 \%$ by the effect of the CP-odd scalars. To obtain the nonzero $g_{\bar{f} f \Delta}$, the scalar fields in the Yukawa terms need to contain CP-odd scalars. For non-vanishing $g_{W W^{\prime} \Delta^{0}}$, the CP-odd scalars have to be components of scalar fields that develop vacuum expectation values (VEVs) because $g_{W W^{\prime} \Delta^{0}}$ originates from kinetic terms of the scalar fields. These two conditions are useful guidelines to construct models that predict $\operatorname{Br}\left(W^{\prime} \rightarrow W Z\right) \gtrsim 2 \%$.

Let us discuss how to construct models that predict $\operatorname{Br}\left(W^{\prime} \rightarrow W Z\right) \gtrsim 2 \%$ by extending HVT model A. It contains two scalar fields $H$ and $\Phi$ which are $(\mathbf{2}, \mathbf{1})_{1 / 2}$ and $(\mathbf{2}, \mathbf{2})_{0}$ under $\mathrm{SU}(2)_{0} \times \mathrm{SU}(2)_{1} \times \mathrm{U}(1)_{2}$, respectively. Since all the $\mathrm{CP}$-odd scalars are eaten by the gauge bosons, we need to add other scalar fields to increase $\Gamma\left(W^{\prime} \rightarrow W Z\right)$. New scalar fields should not have the same gauge charge as $H$ and $\Phi$. If there are more than one scalar fields that have the same gauge charge, we can redefine the scalar fields by taking the linear combination of them and go to a basis where only one of the scalars develops VEV. This is equivalent to add scalars that do not develop VEVs, and thus $g_{W W^{\prime} \Delta^{0}}=0$. A simple choice to obtain nonzero $g_{W W^{\prime} \Delta^{0}}$ is to add a scalar field that is $(\mathbf{1 , 2})_{1 / 2}$. The model with this choice is discussed in Ref. [11, 12] and it certainly predicts large $\operatorname{Br}\left(W^{\prime} \rightarrow W Z\right)$ with appropriate 
parameter choices. Another choice is to add scalar fields that are representations of $\mathrm{SU}(2)_{0}$ and/or $\mathrm{SU}(2)_{1}$ larger than $\mathbf{2}$, for example $(\mathbf{3}, \mathbf{3})_{0}$. However, such scalar fields break the custodial symmetry by their VEVs in many cases, and thus constraints should be studied carefully.

\section{Conclusions}

Spin-1 heavy vector bosons are popular particles predicted in models beyond the SM. They decay into various particles, such as two SM gauge bosons. In this paper, we have investigated the relation between two decay modes, $W^{\prime} \rightarrow W Z$ and $W^{\prime} \rightarrow f f$, from the viewpoint of perturbative unitarity. We have focused on the amplitudes of $f \bar{f} \rightarrow V^{-} V^{+}$where $V=W, W^{\prime}$, and required that these processes do not have bad high-energy behavior at the tree level. This requirement relates the couplings in the amplitudes to each other. The coupling relations obtained from this requirement are called unitarity sum rules.

Utilizing the unitarity sum rules for the system that contains spin-1 heavy vector bosons and $\mathrm{CP}$-even scalars as well as all the SM particles, we have shown that $\Gamma\left(W^{\prime} \rightarrow W Z\right) / \sum_{f} \Gamma\left(W^{\prime} \rightarrow\right.$ $f f) \simeq 1 / 48$ where we sum over the contributions from three generations in both the quark and the lepton sectors. Using this result, we have shown that $\operatorname{Br}\left(W^{\prime} \rightarrow W Z\right) \lesssim 2 \%$ in the system. This result has been derived by imposing perturbative unitarity only on $f \bar{f} \rightarrow W^{-} W^{\prime+}$. The same result is thus obtained even if perturbative unitarity is violated in other processes such as $W W \rightarrow W W$. The result is independent of the number of the CP-even scalars. Moreover, the ratio of the two decay modes is independent of details of models. Hence we conclude that the result can be applied to various models. If $\operatorname{Br}\left(W^{\prime} \rightarrow W Z\right)$ is measured in future and is larger than $2 \%$, then perturbative unitarity requires new particles in addition to $V^{\prime}$ and CP-even scalars.

We have also shown that CP-odd scalars help to increase $\operatorname{Br}\left(W^{\prime} \rightarrow W Z\right)$ if they couple to both the SM fermions and the SM gauge bosons. In contrast to the case without the CP-odd scalars, $\operatorname{Br}\left(W^{\prime} \rightarrow W Z\right)$ depends on the parameters that are determined by the CP-odd scalar couplings and can be much larger than $2 \%$. Depending on the couplings, the decay mode of $W^{\prime}$ to the SM fermions is highly suppressed and the decay mode of $W^{\prime}$ to the gauge bosons can be dominant. This is a big difference of the models with CP-odd scalars from the models only with CP-even scalars. The measurement of decay properties of $W^{\prime}$ is thus important not only for understanding the property of $W^{\prime}$ itself but also for revealing the structure of the system containing $W^{\prime}$. For example, we can estimate the strength of the CP-odd scalar couplings to the SM particles from the measurement of $\operatorname{Br}\left(W^{\prime} \rightarrow e v\right)$ before the discovery of the CP-odd scalars. The result is also useful for model building. For example, we can see that the CP-odd scalars must be components of scalar fields that develop vacuum expectation values in order to obtain large $\operatorname{Br}\left(W^{\prime} \rightarrow W Z\right)$, because the nonzero CP-odd couplings to $W$ and $W^{\prime}$ are required for large $\operatorname{Br}\left(W^{\prime} \rightarrow W Z\right)$ and the couplings arise from the scalar kinetic terms.

\section{Acknowledgments}

This work was supported by JSPS KAKENHI Grant Number 16K17715 [TA] and Research Fellowships of the Japan Society for the Promotion of Science (JSPS) for Young Scientists No. 263947 [RN]. 


\section{References}

[1] I. Low, R. Rattazzi and A. Vichi, JHEP 1004, 126 (2010) doi:10.1007/JHEP04(2010)126 [arXiv:0907.5413 [hep-ph]].

[2] D. Pappadopulo, A. Thamm, R. Torre and A. Wulzer, JHEP 1409, 060 (2014) doi:10.1007/JHEP09(2014)060 [arXiv:1402.4431 [hep-ph]].

[3] G. Aad et al. [ATLAS Collaboration], Phys. Lett. B 737, 223 (2014) doi:10.1016/j.physletb.2014.08.039 [arXiv:1406.4456 [hep-ex]].

[4] V. Khachatryan et al. [CMS Collaboration], Phys. Lett. B 748, 255 (2015) doi:10.1016/j.physletb.2015.07.011 [arXiv:1502.04994 [hep-ex]].

[5] G. Aad et al. [ATLAS Collaboration], Eur. Phys. J. C 75, no. 6, 263 (2015) doi:10.1140/epjc/s10052-015-3474-x [arXiv:1503.08089 [hep-ex]].

[6] V. Khachatryan et al. [CMS Collaboration], JHEP 1602, 145 (2016) doi:10.1007/JHEP02(2016)145 [arXiv:1506.01443 [hep-ex]].

[7] V. Khachatryan et al. [CMS Collaboration], Eur. Phys. J. C 76, no. 5, 237 (2016) doi:10.1140/epjc/s10052-016-4067-z [arXiv:1601.06431 [hep-ex]].

[8] M. Aaboud et al. [ATLAS Collaboration], JHEP 1609, 173 (2016) doi:10.1007/JHEP09(2016)173 [arXiv:1606.04833 [hep-ex]].

[9] G. D’Ambrosio, G. F. Giudice, G. Isidori and A. Strumia, Nucl. Phys. B 645, 155 (2002) doi:10.1016/S0550-3213(02)00836-2 [hep-ph/0207036].

[10] T. Abe, R. Nagai, S. Okawa and M. Tanabashi, Phys. Rev. D 92 (2015) no.5, 055016 doi:10.1103/PhysRevD.92.055016 [arXiv:1507.01185 [hep-ph]].

[11] T. Abe and R. Kitano, Phys. Rev. D 88, no. 1, 015019 (2013) doi:10.1103/PhysRevD.88.015019 [arXiv:1305.2047 [hep-ph]].

[12] T. Abe, T. Kitahara and M. M. Nojiri, JHEP 1602, 084 (2016) doi:10.1007/JHEP02(2016)084 [arXiv:1507.01681 [hep-ph]]. 